\title{
Frequency-dependent lipid raft uptake at rat diaphragm muscle axon terminals
}

\author{
Maria A. Gonzalez Porras, PhD ${ }^{1}$, Matthew J. Fogarty, $\mathrm{PhD}^{1,3}$, Heather M. Gransee, $\mathrm{PhD}^{2}$, \\ Gary C. Sieck, $\mathbf{P h D}^{1,2}$, and Carlos B. Mantilla, MD, $\mathbf{P h D}^{1,2}$ \\ ${ }^{1}$ Department of Physiology \& Biomedical Engineering, Mayo Clinic, Rochester, MN. \\ ${ }^{2}$ Department of Anesthesiology and Perioperative Medicine, Mayo Clinic, Rochester, MN. \\ ${ }^{3}$ School of Biomedical Sciences, The University of Queensland, St Lucia, QLD, Australia
}

\begin{abstract}
Introduction: In motor neurons, cholera toxin B (CTB) binds to the cell-surface ganglioside GM1 and is internalized and transported via structurally unique components of plasma membranes (lipid rafts).

Methods: Lipid raft uptake by axon terminals adjoining type-identified rat diaphragm muscle fibers was investigated using CTB and confocal imaging.

Results: Lipid raft uptake increased significantly at higher-frequency stimulation $(80 \mathrm{~Hz})$, compared to lower-frequency $(20 \mathrm{~Hz})$ and unstimulated $(0 \mathrm{~Hz})$ conditions. The fraction of axon terminal occupied by CTB was $\sim 45 \%$ at 0 or $20 \mathrm{~Hz}$ stimulation, and increased to $\sim 65 \%$ at $80 \mathrm{~Hz}$. Total CTB fluorescence intensity also increased $(20 \%)$ after $80 \mathrm{~Hz}$ stimulation compared to $0 \mathrm{~Hz}$.

Discussion: Evidence of increased lipid raft uptake at high stimulation frequencies supports an important role for lipid raft signaling at rat diaphragm muscle axon terminals, primarily for motor units physiologically activated at the higher frequencies.
\end{abstract}

\section{Keywords}

Neuromuscular junction; Cholera toxin; Bulk endocytosis; Motor neuron; Synaptic vesicle retrieval; Clathrin

\section{Introduction}

\begin{abstract}
Lipid rafts are structurally unique microdomains of plasma membranes, crucial for neural development and function including synaptic transmission and neurotrophic signaling. ${ }^{1}$

These microdomains are enriched in sphingolipids, cholesterol binding proteins and scaffolding proteins. Lipid rafts in motor neurons serve to shuttle cargo between peripheral
\end{abstract}

Corresponding Author: Carlos B. Mantilla, MD, PhD, MB 2-758, St Mary's Hospital, Mayo Clinic, 200 First St SW, Rochester, MN 55905, Tel: (507) 255-7481, Fax: (507) 255-7300, mantilla.carlos@ mayo.edu.

None of the authors has any conflict of interest to disclose

We confirm that we have read the Journal's position on issues involved in ethical publication and affirm that this report is consistent with those guidelines 
and central nervous system compartments, ${ }^{2}$ but their dynamics at axon terminals is poorly characterized. Motor units differ in their synaptic properties ${ }^{3-7}$ and their activation pattern, ${ }^{8}$ such that lipid raft uptake may differ across axon terminals adjoining the different muscle fiber types as well as the motor unit activation frequencies. Motor units can be classified as fatigue resistant motor units (slow-twitch type $S$ and fast-twitch type FR units) or fatigable motor units (fast-twitch type FInt and type FF units), which innervate type I or type IIa muscle fibers vs. type IIx and/or IIb fibers, respectively. ${ }^{9-11}$ The activation (recruitment) of motor units occurs in an orderly fashion based on the intrinsic electrophysiological properties of their constituent motor neuron. ${ }^{12}$ For instance, in muscles of mixed fiber type composition, like the diaphragm muscle, type $\mathrm{S}$ and FR motor units are recruited before and more often than type FInt or type FF units. ${ }^{13-15}$ Activation frequencies also vary across motor units with higher frequencies at more fatigable units. ${ }^{8}$

Lipid rafts are readily labeled by the atoxic subunit B of cholera toxin (CTB), and CTB is commonly used to investigate lipid raft function within neurons. ${ }^{16}$ The CTB subunit binds to a cell-surface receptor, ganglioside GM1, present on neuronal membranes, ${ }^{17,18}$ is internalized and then transported via lipid rafts to various neuronal compartments. Axon terminals display substantial membrane cycling, in an activity-dependent fashion, ${ }^{19,20}$ and endocytotic mechanisms responsible for membrane recycling and retrieval may depend on axon terminal activity. Clathrin-mediated endocytosis is sufficient for membrane retrieval when fewer synaptic vesicle are released, i.e., following activation at lower stimulation frequencies. ${ }^{21,22}$ Since the maximal rate of clathrin-mediated endocytosis does not scale with stimulation intensity, ${ }^{23-25}$ when more synaptic vesicle membrane requires retrieval (i.e., following higher frequency stimulation), additional endocytotic mechanisms (e.g., bulk endocytosis) become necessary. ${ }^{20,26,27}$ The fact that bulk endocytosis is not specific for recovering synaptic vesicle endocytosis led us to hypothesize that lipid raft uptake at diaphragm muscle axon terminals depends on activation frequency, such that lipid raft uptake will only be evident at higher stimulation frequencies.

\section{Methods}

\section{Animals:}

Young (6-months old) female and male Fischer 344 pathogen-free rats were obtained from the NIH aged rodent colony. All experiments were approved by the Mayo Clinic Institutional Animal Care and Use Committee. Following deep ketamine $(80 \mathrm{mg} / \mathrm{kg})$ and xylazine $(20 \mathrm{mg} / \mathrm{kg})$ intramuscular anesthesia, rats were killed by exsanguination.

\section{Diaphragm muscle phrenic nerve preparations:}

The diaphragm muscle with the phrenic nerves attached was dissected, and placed in oxygenated Rees-Simpson solution (containing, in mM: $135 \mathrm{Na}^{+}, 5 \mathrm{~K}^{+}, 2 \mathrm{Ca}^{2+}, 1 \mathrm{Mg}^{2+}, 120$ $\mathrm{Cl}^{-}$, and $25 \mathrm{HCO}_{3}^{-} ; \mathrm{pH}$ 7.4) bubbled with carbogen gas $\left(95 \% \mathrm{O}_{2}-5 \% \mathrm{CO}_{2}\right)$ at room temperature $\left(26^{\circ} \mathrm{C}\right)$. The muscle was cut into two hemidiaphragms and pinned in a silicon rubber (Sylgard; DowCorning, Midland, MI)-coated dish at 1.5 times resting length (approximate optimal length for force generation). $10 \mu \mathrm{g}$ of Alexa-488 conjugated CTB (Thermo Fisher Scientific, Waltham, MA) was added to the bath with one of the 
hemidiaphragms not stimulated and serving as an internal control. The other hemidiaphragm was repeatedly activated via phrenic nerve stimulation using a suction electrode with stimulation driven by an Aurora high-power biphasic stimulator (Aurora, ON, Canada). Supramaximal stimulation was presented in $0.5 \mathrm{~ms}$ duration pulses delivered either at $20 \mathrm{~Hz}$ or $80 \mathrm{~Hz}$. Stimulation at $20 \mathrm{~Hz}$ was delivered in $330 \mathrm{~ms}$ duration trains repeated each s $(33 \%$ duty cycle) for $1 \mathrm{~h}$ in order to achieve physiological activation levels for rat phrenic motor units. $^{8,28}$ Stimulation at $80 \mathrm{~Hz}$ was applied in $1 \mathrm{~s}$ duration trains repeated every other s $(50 \%$ duty cycle) for $6 \mathrm{~min}$ followed by a $2 \mathrm{~min}$ incubation period in the presence of CTB without stimulation to allow slow endocytosis mechanisms to be completed and in order to elicit reliable bulk endocytosis at this higher frequency. ${ }^{29}$ Visible contractions of the diaphragm muscle served to verify successful nerve stimulation in all cases.

Each hemidiaphragm was then fixed in $4 \%$ paraformaldehyde and incubated with abungarotoxin conjugated to Alexa Fluor 555 (0.1 $\mu \mathrm{g} / \mathrm{ml}$; Catalog\#: B35451 \& Lot\# 1880574. Invitrogen Corp. Carlsbad CA) to stain the postsynaptic motor end-plate. Antisynaptophysin antibody D-4 was used to stain the presynaptic terminals $(1 \mathrm{mg} / \mathrm{ml}$; Catalog\#: sc17750 \& Lot\# 135090. Santa Cruz Biotechnology Inc., Santa Cruz, CA) with an Alexa 647 conjugated donkey anti-mouse IgG secondary antibody (1:200; Catalog\#: 715-605-159 \& Lot\# 135090. Jackson Immuno Research Laboratories Inc., West Grove, PA). All diaphragm muscle samples were stored in Tris-buffered saline until confocal imaging, which occurred no more than 3 days following the staining procedure. The specificity of fluorescently-conjugated a-bungarotoxin and anti-synaptophysin labeling has been previously confirmed and validated. 30,7

\section{Confocal imaging:}

Confocal microscopy was used for morphological analyses of neuromuscular junction (NMJ) morphology, as previously described, ${ }^{5,31-33}$ and measurements of CTB uptake in diaphragm axon terminals adjoining type-identified muscle fibers. Motor end-plates labeled by Alexa Fluor 555-a-bungarotoxin and Alexa-488 conjugated CTB were imaged simultaneously in two channels, with Alexa 647-labeled axon terminals (synaptophysin immunoreactivity) imaged in series. Confocal image stacks were obtained from NMJs visualized en face on the thoracic surface of the diaphragm muscle and located superficially (usually within $50 \mu \mathrm{m}$ of the surface) in order to minimize fluorescence dispersion and to reduce the impact of anisotropic voxel dimensions on image reconstruction. Superficially located NMJs are not morphologically different from other NMJs located at greater depths in midcostal region of the rat diaphragm muscle. $4,34,7,3$

\section{Neuromuscular junction morphology:}

Diaphragm NMJs were identified at type I or IIa or type IIx and/or IIb muscle fibers based on well-established morphological criteria. ${ }^{7,3}$ Diaphragm NMJs at type I or IIa fibers are smaller and less complex that those at type IIx and/or IIb fibers. This user-guidance classification system has been validated and used extensively for diaphragm NMJs. 6,5,3,35,34,36 Two-dimensional projections for each motor end-plate were obtained using a maximum intensity projection algorithm available in MetaMorph (Molecular Devices, San Jose, CA) software. The relative planar area occupied by the motor end-plate was measured 
as an index of NMJ complexity that reflects branching and fragmentation (greater branching and fragmentation results in reduced relative planar area).

\section{CTB uptake measurements:}

CTB fluorescence intensity in the presynaptic volume was measured for each NMJ, using a customized algorithm in MetaMorph. ${ }^{37,38}$ In NMJs exhibiting colocalized apposition of synaptophysin and a-bungarotoxin, the volume of the axon terminal occupied by CTB was determined from the intersection of the two binarized volumes in 3D. In addition, the percentage of axon terminals with robust CTB uptake (i.e., intensity $\sim 10$-fold greater than background) was assessed. In a separate experiment, the total summed Alexa 488-CTB fluorescence intensity per axon terminal was obtained after thresholding to subtract tissue auto-fluorescence and normalized to the motor end plate area (a-bungarotoxin maximum intensity projection).

\section{Statistical methods:}

Individual NMJs were analyzed from all animals and were not clustered by animal in order to account for variability across NMJs. In all cases, there was no animal effect. All data sets were assessed for normality using Shapiro-Wilk W-tests. Where data was distributed normally, comparisons of lipid raft uptake according to muscle fiber type classification (type I or type IIa Vs. type IIx and/or IIb) and stimulation frequency $(0,20$ and $80 \mathrm{~Hz})$, were done using two-way ANOVA with Tukey-Kramer HSD post-tests as appropriate. Significance was set at $\mathrm{P}<0.05$ and all data are presented as mean \pm standard deviation, unless otherwise specified. For data where distributions were not normally distributed (CTB summed intensity), nonparametric Wilcoxon comparisons were employed.

\section{Results}

\section{Morphological differences across NMJs at different diaphragm muscle fiber types}

Motor end-plates and axon terminals were labeled using fluorescently conjugated abungarotoxin and anti-synaptophysin, respectively (Figure 1). The NMJ planar area was used as a criterion to classify the NMJs in the two fiber type classes. Motor end-plate areas at type I or IIa fibers were comparable in size, and $~ 50 \%$ smaller $\left(489 \pm 130 \mu \mathrm{m}^{2}\right)$ than motor end-plate areas at type IIx and/or IIb fibers $\left(1095 \pm 380 \mu \mathrm{m}^{2} ; \mathrm{F}_{(1,206)}=229, P<\right.$ 0.001 ). The complexity index was used to measure branching and fragmentation in the motor end plate area and is a function of relative planar area compared to the orthogonal area circumscribing the end plate. Motor end-plates at type I or IIa fibers were significantly less complex (greater relative planar area) than those at type IIx and/or IIb fibers. In type IIx and/or IIb fibers, relative planar area was $52 \pm 8 \%$ compared to $56 \pm 11 \%$ in type I or type IIa fibers $(\mathrm{F}(1,206)=4.8, \mathrm{P}=0.03 ; \mathrm{n}=207$ from 7 animals). Pre-synaptic volume is also $\sim 2$ fold larger at type IIx and/or IIb fibers $\left(6,003 \pm 2,500 \mu \mathrm{m}^{3}\right)$, compared to type I or type IIa fibers $\left(2,951 \pm 1,200 \mu \mathrm{m}^{3} ; \mathrm{F}_{(1,206)}=120, \mathrm{P}<0.0001\right.$; Figure 2).

\section{Lipid raft uptake at diaphragm axon terminals in unstimulated conditions}

Different characteristics were evaluated to analyze changes in lipid raft uptake in diaphragm muscle axon terminals. First, the CTB volume in pre-synaptic terminals was compared 
across axon terminals adjoining type I or IIa muscle fibers compared to those adjoining type IIx and/or IIb fibers. The CTB volume in terminals adjoining type I or IIa muscle fibers was $1658 \pm 520 \mu \mathrm{m}^{3}$, and for IIx and/or IIb terminals was $3965 \pm 1529 \mu \mathrm{m}^{3}\left(\mathrm{~F}_{(1,84)}=94\right.$, $\mathrm{P}<0.0001)$. Although the overall CTB volume is greater in axon terminals adjoining type IIx and/or IIb diaphragm muscle fibers, when normalized to the volume of the respective axon terminal, this difference in CTB volume across fiber type disappears. The percent of the axon terminal occupied by CTB, determined by the fraction of axon terminal volume (labeled by synaptophysin) showing colocalization with CTB, was compared across axon terminals classified according to fiber type (Figure 3 ). There was no difference in the fraction of axon terminal occupied by CTB at both types of NMJs $(\mathrm{P}=0.26)$. These results demonstrate that the distribution of lipid rafts and their basal level of uptake at the NMJ are not different across fiber types. Observed differences in CTB volumes were a result of differences in NMJ size.

\section{Frequency dependent uptake of CTB at axon terminals across fiber types}

To analyze stimulation dependent changes in lipid raft uptake at axon terminals, the CTB volume and percent of NMJs showing CTB uptake were used. First, the CTB volume in presynaptic terminals was compared with and without low frequency stimulation $(20 \mathrm{~Hz})$, at which both fatigue-resistant and more fatigable motor units are activated (Figure 3 ). The fraction of axon terminal occupied by CTB with no stimulation and at $20 \mathrm{~Hz}$ stimulation was $\sim 45 \%$ across all muscle fiber types $(\mathrm{P}=0.45)$. These results demonstrate that lipid raft uptake does not change between unstimulated and low frequency stimulation conditions.

Effective uptake of CTB (i.e., the \% of NMJs with evidence of CTB within axon terminals), into both type I or IIa and type IIx and/or IIb NMJs was apparent at both 0 and $20 \mathrm{~Hz}$ stimulation frequencies (range: 91-95\%). There was no evidence of an effect on the proportion of axon terminals displaying lipid raft uptake according to fiber type $\left(\mathrm{F}_{(1,36)}=\right.$ $0.9, P=0.34)$, stimulation frequency $\left(\mathrm{F}_{(2,36)}=1.4, P=0.27\right)$ or the interaction $\left(\mathrm{F}_{(2,36)}=0.2\right.$, $P=0.85)$.

\section{Lipid raft uptake at high frequency stimulation}

To activate bulk endocytosis mechanisms, we used high frequency stimulation at $80 \mathrm{~Hz}$ in 1 $\mathrm{s}$ duration trains repeated every other s (50\% duty cycle) for $6 \mathrm{~min}$ followed by a $2 \mathrm{~min}$ period of incubation in CTB without stimulation. From each animal, one hemidiaphragm was not stimulated and served as an internal time control for CTB uptake. Compared to control unstimulated NMJs ( $N=7$ animals) and to NMJs stimulated at $20 \mathrm{~Hz}(\mathrm{~N}=4$ animals), the fraction of the axon terminal occupied by lipid rafts (CTB) increased significantly in presynaptic terminals after $80 \mathrm{~Hz}$ stimulation ( $\mathrm{N}=3$ animals) at both type I or IIa and type IIx and/or IIb muscle fibers (from $\sim 45 \%$ to $\sim 65 \%$ of presynaptic volume occupied by CTB; Figure 3).

In an effort to further understand the uptake of lipid rafts in axon terminals at a higher stimulation frequency, total CTB fluorescence intensity was quantified across presynaptic terminals adjoining the different muscle fiber types (Figure 4). Such quantitative measurements were permitted by the use of fluorescently conjugated CTB with the sum of 
CTB fluorescence intensity at each type-identified axon terminal reflecting uptake of CTB bound to lipid rafts. There was a significant increase in the CTB intensity in NMJs at type I or IIa diaphragm fibers $(\mathrm{P}=0.012)$ and at type IIx and/or IIb fibers $(\mathrm{P}=0.003)$ stimulated with high frequency compared to unstimulated NMJs from the same type (Figure 4). The increased CTB fluorescence intensity observed at this high frequency stimulation represents an increase of lipid raft enriched vesicles, as observed in the CTB images (Figure 4).

\section{Discussion}

Our results demonstrate that lipid raft uptake is evident across axon terminals adjoining different fiber types in the rat diaphragm muscle at rest and during low frequency nerve stimulation, consistent with clathrin-dependent synaptic vesicle endocytosis. Lipid raft uptake increases after nerve stimulation at higher frequencies, consistent with different endocytic mechanisms being involved, namely bulk endocytosis. ${ }^{27,29}$ Recruitment of fatigue-resistant motor units (comprising type I and IIa muscle fibers), ${ }^{8}$ which discharge physiologically at low frequencies $(\sim 8-25 \mathrm{~Hz}), 39,13,40,41$ is sufficient to generate the lower forces displayed during eupneic ventilation and in response to hypoxic-hypercapnic exposure. The generation of higher forces involved in expulsive behaviors and airway clearance (e.g., coughing, sneezing) requires recruitment of more fatigable motor units (comprising type IIx and/or IIb muscle fibers) that discharge at higher frequencies. 42,15,43,44,14 Evidence of increased lipid raft uptake at high stimulation frequencies supports the importance of lipid raft signaling in motor units that are physiologically activated at the higher frequencies (i.e., those innervating type IIx and/or IIb muscle fibers).

\section{Lipid raft presence in axon terminals}

In neurons, lipid rafts compartmentalize transmembrane neurotransmitter receptors and necessary intracellular signaling adaptor proteins, ${ }^{45-47}$ contribute to the trafficking of components to and from the cell membrane, and participate in organelle trafficking. ${ }^{4}$ Accordingly, lipid rafts are expected to be present throughout the neuronal membrane including axon terminals, and in the present study, we observed evidence of lipid raft labeling by CTB across type-identified diaphragm muscle fibers. The morphological characteristics of NMJs at type-identified diaphragm muscle fibers was consistent with previous reports, ${ }^{3,7}$ serving as the basis for fiber type classification. In unstimulated conditions, the relative lipid raft density was not different across type I or IIa diaphragm muscle fibers compared to type IIx and/or IIb fibers, consistent with small differences in membrane recycling (e.g., reflected by miniature end plate potentials, mEPP). ${ }^{6}$ Notably, mEPP frequency increases just $\sim 1 \mathrm{~Hz}$ from room to body temperature, and the quantal content is quite insensitive to temperature differences. ${ }^{49,50}$ Our results from studies conducted at room temperature support a role for lipid raft dynamics (uptake and transport) in modulating intracellular signaling events, trophic interactions and motor neuron function.

\section{Lipid raft uptake across axon terminals adjoining different fiber types}

Lipid raft uptake increased at higher stimulation frequencies in axon terminals across rat diaphragm muscle fibers. Although there were no differences in relative lipid raft uptake in axon terminals adjoining both type I or IIa vs. type IIx and/or IIb diaphragm muscle fibers, 
there are known differences in motor unit recruitment and activation history across motor units of different type. Motor units comprising type I or IIa fibers are recruited more frequently with lower activation rates ( $\sim 20 \mathrm{~Hz}$ for ventilatory behaviors) than units comprising type IIx and/or IIb fibers, which are infrequently recruited and only for short durations (e.g., for expulsive behaviors). ${ }^{42,14,43,44}$ The release and recycling of synaptic vesicles is stimulation frequency dependent and varies across motor unit types in the diaphragm muscle. ${ }^{6,51}$

The results of the present study are consistent with a lack of involvement of lipid rafts in the endocytic mechanisms responsible for synaptic vesicle retrieval at lower activation rates. Previous studies support the premise that synaptic vesicle recycling at low physiological frequencies depends on clathrin-mediated endocytosis, which facilitates retrieval of single synaptic vesicles from the axon terminal plasma membrane. ${ }^{19,27}$ Since type I or IIa fibers in the rat diaphragm muscle display peak activation frequencies $\sim 20 \mathrm{~Hz},{ }^{8}$ the results of the present study suggest that clathrin-mediated endocytosis does not contribute to the uptake of membrane compartments that include lipid rafts. Although at body temperature the speed of clathrin-mediated endocytosis is faster compared to room temperature, ${ }^{52,53}$ the magnitude of this change is small relative to the effect on lipid raft uptake observed at higher stimulation frequencies.

\section{Endocytosis of lipid rafts at higher stimulation frequencies requiring bulk endocytosis}

During increased neuronal activity, additional synaptic vesicle retrieval capacity is required and is provided by bulk endocytosis. ${ }^{54,20}$ Since bulk endocytosis can internalize large areas of plasma membrane, it has the potential capacity to correct for significant changes in axon terminal surface area during increased neuronal activity. ${ }^{55}$ In the present study, the uptake of lipid rafts after $80 \mathrm{~Hz}$ stimulation increased at axon terminals adjoining all diaphragm muscle fiber types compared to unstimulated conditions, suggesting that endosomes generated from bulk endocytosis at axon terminals contain lipid rafts. These findings in the rat diaphragm muscle are consistent with previous studies demonstrating that a number of fluid phase uptake routes related to bulk endocytosis have an enrichment of lipid rafts. ${ }^{20,56}$ Bulk endosomes help in recovering synaptic transmission (recycled into plasma membrane $)^{57}$ but they can also participate in retrograde and other intracellular signaling events, ${ }^{58-60}$ suggesting that motor neurons discharging at high frequencies rely on lipid raft endocytosis for activity dependent intracellular signaling.

The maturation process to obtain synaptic vesicles after bulk endocytosis occurs after the end of the stimulation and may contribute to physiological differences in motor unit properties. ${ }^{57}$ Limits in the ability to obtain synaptic vesicles from bulk endocytosis may partially underlie the increased likelihood of neuromuscular transmission failure of axon terminals innervating type IIx and/or IIb muscle fibers and their decreased safety factor during repeated high frequency stimulation. ${ }^{61,6,62}$ The role that lipid rafts exert in regulating such bulk endocytic mechanisms remains unclear. Since stimulation frequencies that result in bulk endocytosis are more commonly present at the most fatigable motor units (innervating type IIx and/or IIb fibers), presumably these units are more susceptible to activity dependent differences in lipid raft signaling. Whether activity dependent differences 
in lipid raft dependent signaling (including retrograde trophic effects) contribute to motor neuron health or disease is an important topic for future study.

\section{Conclusions and Future Directions}

Lipid rafts form domains for assembly of multiple signaling complexes, and thus, changes in lipid raft uptake may modulate intracellular signaling pathways. In particular, motor units comprising type IIx and/or IIb muscle fibers could be more susceptible to changes in lipid raft uptake, since their discharge rate is characterized by high frequencies. These results have intriguing implications for neurodegenerative diseases, aging, synaptic plasticity and other conditions where deficits in lipid raft trafficking may render specific neuronal populations susceptible to injury as a result of disrupted trophic signaling. ${ }^{34,63}$ If lipid rafts contribute to synaptic stability (e.g., preventing denervation) as proposed for other neuron types, ${ }^{64-66}$ novel therapies that address the mechanisms responsible for disrupted lipid rafts or that promote membrane lipid raft formation and trafficking in motor neurons could offer promising therapeutic targets across a range of conditions. It is important to note that conclusions of the study are based on results obtained in the diaphragm muscle. These results are the first step in understanding the role of lipid rafts in neuromuscular diseases and also providing a new way of targeting different motor neurons at the NMJ through specific endocytic mechanisms.

\section{Acknowledgements:}

This work was supported by National Institutes of Health Grants AG-057052, AG-044615, HL-096750. (M. A. Gonzalez Porras, G. C. Sieck and C. B. Mantilla) and an Australian National Health and Medical Research Council CJ Martin Early Career Fellowship (M. J. Fogarty).

\section{Abbreviations: \\ CTB \\ cholera toxin subunit B \\ NMJ \\ neuromuscular junction}

\section{References}

1. Egawa J, Pearn ML, Lemkuil BP, Patel PM, Head BP. Membrane lipid rafts and neurobiology: agerelated changes in membrane lipids and loss of neuronal function. J Physiol 2016;594(16):45654579. [PubMed: 26332795]

2. Wang T, Martin S, Nguyen TH, Harper CB, Gormal RS, Martinez-Marmol R, Karunanithi S, Coulson EJ, Glass NR, Cooper-White JJ, van Swinderen B, Meunier FA. Flux of signalling endosomes undergoing axonal retrograde transport is encoded by presynaptic activity and TrkB (vol 7, 12976, 2016). Nat Commun 2016;7.

3. Mantilla CB, Rowley KL, Fahim MA, Zhan WZ, Sieck GC. Synaptic vesicle cycling at typeidentified diaphragm neuromuscular junctions. Muscle Nerve 2004;30(6):774-783. [PubMed: 15478121]

4. Prakash YS, Miller SM, Huang M, Sieck GC. Morphology of diaphragm neuromuscular junctions on different fibre types. Journal of Neurocytology 1996;25(2):88-100. [PubMed: 8699198]

5. Mantilla CB, Rowley KL, Zhan WZ, Fahim MA, Sieck GC. Synaptic vesicle pools at diaphragm neuromuscular junctions vary with motoneuron soma, not axon terminal, inactivity. Neuroscience 2007;146(1):178-189. [PubMed: 17346898] 
6. Rowley KL, Mantilla CB, Ermilov LG, Sieck GC. Synaptic vesicle distribution and release at rat diaphragm neuromuscular junctions. J Neurophysiol 2007;98(1):478-487. [PubMed: 17493926]

7. Sieck DC, Zhan WZ, Fang YH, Ermilov LG, Sieck GC, Mantilla CB. Structure-activity relationships in rodent diaphragm muscle fibers vs. neuromuscular junctions. Respir Physiol Neurobiol 2012;180(1):88-96. [PubMed: 22063925]

8. Seven YB, Mantilla CB, Sieck GC. Recruitment of Rat Diaphragm Motor Units Across Motor Behaviors with Different Levels of Diaphragm Activation. J Appl Physiol 2014;117(11):1308-1316. [PubMed: 25257864]

9. Fournier M, Sieck GC. Mechanical properties of muscle units in the cat diaphragm. J Neurophysiol 1988;59(3):1055-1066. [PubMed: 3367195]

10. Sieck GC. Diaphragm muscle: structural and functional organization. Clin Chest Med 1988;9(2): 195-210. [PubMed: 3292123]

11. Sieck GC, Fournier M, Enad JG. Fiber type composition of muscle units in the cat diaphragm. Neuroscience Letters 1989;97(1-2):29-34. [PubMed: 2521928]

12. Henneman E, Somjen G, Carpenter DO. Functional significance of cell size in spinal motoneurons. J Neurophysiol 1965;28(3):560-580. [PubMed: 14328454]

13. Butler JE, McKenzie DK, Gandevia SC. Discharge properties and recruitment of human diaphragmatic motor units during voluntary inspiratory tasks. J Physiol 1999;518 (Pt 3):907-920. [PubMed: 10420024]

14. Sieck GC, Fournier M. Diaphragm motor unit recruitment during ventilatory and nonventilatory behaviors. J Appl Physiol 1989;66(6):2539-2545. [PubMed: 2745316]

15. Mantilla CB, Sieck GC. Phrenic motor unit recruitment during ventilatory and non-ventilatory behaviors. Respir Physiol Neurobiol 2011;179(1):57-63. [PubMed: 21763470]

16. Head BP, Hu Y, Finley JC, Saldana MD, Bonds JA, Miyanohara A, Niesman IR, Ali SS, Murray F, Insel PA, Roth DM, Patel HH, Patel PM. Neuron-targeted caveolin-1 protein enhances signaling and promotes arborization of primary neurons. J Biol Chem 2011;286(38):33310-33321. [PubMed: 21799010]

17. Sheikh KA, Deerinck TJ, Ellisman MH, Griffin JW. The distribution of ganglioside-like moieties in peripheral nerves. Brain 1999;122 (Pt 3):449-460. [PubMed: 10094254]

18. Zhang RG, Westbrook ML, Westbrook EM, Scott DL, Otwinowski Z, Maulik PR, Reed RA, Shipley GG. The 2.4 A crystal structure of cholera toxin B subunit pentamer: choleragenoid. J Mol Biol 1995;251(4):550-562. [PubMed: 7658472]

19. Royle SJ, Lagnado L. Clathrin-Mediated Endocytosis at the Synaptic Terminal: Bridging the Gap Between Physiology and Molecules. Traffic 2010;11(12):1489-1497. [PubMed: 20633242]

20. Clayton EL, Cousin MA. The molecular physiology of activity-dependent bulk endocytosis of synaptic vesicles. J Neurochem 2009;111(4):901-914. [PubMed: 19765184]

21. Heuser JE, Reese TS. Evidence for recycling of synaptic vesicle membrane during transmitter release at the frog neuromuscular junction. J Cell Biol 1973;57(2):315-344. [PubMed: 4348786]

22. Brodin L, Low P, Shupliakov O. Sequential steps in clathrin-mediated synaptic vesicle endocytosis. Current Opinion in Neurobiology 2000;10(3):312-320. [PubMed: 10851177]

23. Balaji J, Ryan TA. Single-vesicle imaging reveals that synaptic vesicle exocytosis and endocytosis are coupled by a single stochastic mode. Proc Natl Acad Sci U S A 2007;104(51):20576-20581. [PubMed: 18077369]

24. Granseth B, Odermatt B, Royle SJ, Lagnado L. Clathrin-mediated endocytosis is the dominant mechanism of vesicle retrieval at hippocampal synapses. Neuron 2006;51(6):773-786. [PubMed: 16982422]

25. Jockusch WJ, Praefcke GJ, McMahon HT, Lagnado L. Clathrin-dependent and clathrinindependent retrieval of synaptic vesicles in retinal bipolar cells. Neuron 2005;46(6):869-878. [PubMed: 15953416]

26. Clayton EL, Evans GJ, Cousin MA. Bulk synaptic vesicle endocytosis is rapidly triggered during strong stimulation. J Neurosci 2008;28(26):6627-6632. [PubMed: 18579735]

27. Soykan T, Maritzen T, Haucke V. Modes and mechanisms of synaptic vesicle recycling. Current Opinion in Neurobiology 2016;39:17-23. [PubMed: 27016897] 
28. Mantilla CB, Seven YB, Sieck GC. Convergence of pattern generator outputs on a common mechanism of diaphragm motor unit recruitment. Prog Brain Res 2014;209:309-329. [PubMed: 24746055]

29. Maeno-Hikichi Y, Polo-Parada L, Kastanenka KV, Landmesser LT. Frequency-Dependent Modes of Synaptic Vesicle Endocytosis and Exocytosis at Adult Mouse Neuromuscular Junctions. Journal of Neuroscience 2011;31(3):1093-1105. [PubMed: 21248134]

30. Issa AN, Zhan WZ, Sieck G, Mantilla CB. Neuregulin-1 at synapses on phrenic motoneurons. J Comp Neurol 2010;518(20):4213-4225. [PubMed: 20878784]

31. Prakash YS, Smithson KG, Sieck GC. Measurements of motoneuron somal volumes using laser confocal microscopy: comparisons with shape-based stereological estimations. Neuroimage 1993;1(2):95-107. [PubMed: 9343561]

32. Prakash YS, Smithson KG, Sieck GC. Growth-related adaptations of endplates on diaphragm muscle fibers: A 3D study using confocal microscopy. Soc Neurosci Abstr 1994;20:1599.

33. Sieck GC, Mantilla CB, Prakash YS. Volume measurements in confocal microscopy. Methods Enzymol 1999;307:296-315. [PubMed: 10506980]

34. Prakash YS, Sieck GC. Age-related remodeling of neuromuscular junctions on type-identified diaphragm fibers. Muscle Nerve 1998;21(7):887-895. [PubMed: 9626248]

35. Prakash YS, Miyata H, Zhan WZ, Sieck GC. Inactivity-induced remodeling of neuromuscular junctions in rat diaphragmatic muscle. Muscle Nerve 1999;22(3):307-319. [PubMed: 10086891]

36. Sieck GC, Van Balkom RH, Prakash YS, Zhan WZ, Dekhuijzen PN. Corticosteroid effects on diaphragm neuromuscular junctions. J Appl Physiol 1999;86(1):114-122. [PubMed: 9887121]

37. Greising SM, Stowe JM, Sieck GC, Mantilla CB. Role of TrkB kinase activity in aging diaphragm neuromuscular junctions. Experimental Gerontology 2015;72:184-191. [PubMed: 26517952]

38. Mantilla CB, Stowe JM, Sieck DC, Ermilov LG, Greising SM, Zhang C, Shokat KM, Sieck GC. TrkB Kinase Activity Maintains Synaptic Function and Structural Integrity at Adult Neuromuscular Junctions. J Appl Physiol 2014;117(8):910-920. [PubMed: 25170066]

39. Seven YB, Mantilla CB, Zhan WZ, Sieck GC. Non-stationarity and power spectral shifts in EMG activity reflect motor unit recruitment in rat diaphragm muscle. Respir Physiol Neurobiol 2013;185(2):400-409. [PubMed: 22986086]

40. Kong FJ, Berger AJ. Firing properties and hypercapnic responses of single phrenic motor axons in the rat. J Appl Physiol 1986;61(6):1999-2004. [PubMed: 3027021]

41. Iscoe S, Dankoff J, Migicovsky R, Polosa C. Recruitment and discharge frequency of phrenic motoneurones during inspiration. Respir Physiol Neurobiol 1976;26(1):113-128.

42. Mantilla CB, Seven YB, Zhan WZ, Sieck GC. Diaphragm motor unit recruitment in rats. Respir Physiol Neurobiol 2010;173(1):101-106. [PubMed: 20620243]

43. Sieck GC. Neural control of the inspiratory pump. NIPS 1991;6:260-264.

44. Sieck GC. Physiological effects of diaphragm muscle denervation and disuse. Clin Chest Med 1994;15(4):641-659. [PubMed: 7867280]

45. Allen JA, Halverson-Tamboli RA, Rasenick MM. Lipid raft microdomains and neurotransmitter signalling. Nat Rev Neurosci 2007;8(2):128-140. [PubMed: 17195035]

46. Simons K, Toomre D. Lipid rafts and signal transduction. Nat Rev Mol Cell Biol 2000;1(1):31-39. [PubMed: 11413487]

47. Suzuki S, Numakawa T, Shimazu K, Koshimizu H, Hara T, Hatanaka H, Mei L, Lu B, Kojima M. BDNF-induced recruitment of TrkB receptor into neuronal lipid rafts: roles in synaptic modulation. Journal of Cell Biology 2004;167(6):1205-1215. [PubMed: 15596541]

48. Tsui-Pierchala BA, Encinas M, Milbrandt J, Johnson EM, Jr. Lipid rafts in neuronal signaling and function. Trends Neurosci 2002;25(8):412-417. [PubMed: 12127758]

49. Glavinovic MI. Voltage clamping of unparalysed cut rat diaphragm for study of transmitter release. J Physiol 1979;290(2):467-480. [PubMed: 224172]

50. Liley AW. An investigation of spontaneous activity at the neuromuscular junction of the rat. J Physiol 1956;132(3):650-666. [PubMed: 13332600]

51. Kononenko NL, Haucke V. Molecular mechanisms of presynaptic membrane retrieval and synaptic vesicle reformation. Neuron 2015;85(3):484-496. [PubMed: 25654254] 
52. Renden R, von Gersdorff H. Synaptic vesicle endocytosis at a CNS nerve terminal: faster kinetics at physiological temperatures and increased endocytotic capacity during maturation. $\mathrm{J}$ Neurophysiol 2007;98(6):3349-3359. [PubMed: 17942618]

53. Smith SM, Renden R, von Gersdorff H. Synaptic vesicle endocytosis: fast and slow modes of membrane retrieval. Trends Neurosci 2008;31(11):559-568. [PubMed: 18817990]

54. Clayton EL, Evans GJ, Cousin MA. Activity-dependent control of bulk endocytosis by protein dephosphorylation in central nerve terminals. J Physiol 2007;585(Pt 3):687-691. [PubMed: 17584836]

55. Cousin MA. Activity-dependent bulk synaptic vesicle endocytosis--a fast, high capacity membrane retrieval mechanism. Mol Neurobiol 2009;39(3):185-189. [PubMed: 19266323]

56. Doherty GJ, McMahon HT. Mechanisms of endocytosis. Annu Rev Biochem 2009;78:857-902. [PubMed: 19317650]

57. Nguyen TH, Maucort G, Sullivan RK, Schenning M, Lavidis NA, McCluskey A, Robinson PJ, Meunier FA. Actin- and dynamin-dependent maturation of bulk endocytosis restores neurotransmission following synaptic depletion. PloS one 2012;7(5):e36913. [PubMed: 22629340]

58. Schmieg N, Menendez G, Schiavo G, Terenzio M. Signalling endosomes in axonal transport: travel updates on the molecular highway. Semin Cell Dev Biol 2014;27:32-43. [PubMed: 24171925]

59. Deinhardt K, Salinas S, Verastegui C, Watson R, Worth D, Hanrahan S, Bucci C, Schiavo G. Rab5 and Rab7 control endocytic sorting along the axonal retrograde transport pathway. Neuron 2006;52(2):293-305. [PubMed: 17046692]

60. Wang T, Martin S, Papadopulos A, Harper CB, Mavlyutov TA, Niranjan D, Glass NR, CooperWhite JJ, Sibarita JB, Choquet D, Davletov B, Meunier FA. Control of autophagosome axonal retrograde flux by presynaptic activity unveiled using botulinum neurotoxin type a. J Neurosci 2015;35(15):6179-6194. [PubMed: 25878289]

61. Johnson BD, Sieck GC. Differential susceptibility of diaphragm muscle fibers to neuromuscular transmission failure. J Appl Physiol 1993;75(1):341-348. [PubMed: 8397179]

62. Ermilov LG, Mantilla CB, Rowley KL, Sieck GC. Safety factor for neuromuscular transmission at type-identified diaphragm fibers. Muscle Nerve 2007;35(6):800-803. [PubMed: 17286272]

63. Fogarty MJ, Omar TS, Zhan WZ, Mantilla CB, Sieck GC. Phrenic Motor Neuron Loss in Aged Rats. J Neurophysiol 2018;119(5):1852-1862. [PubMed: 29412773]

64. Schilling JM, Cui W, Godoy JC, Risbrough VB, Niesman IR, Roth DM, Patel PM, Drummond JC, Patel HH, Zemljic-Harpf AE, Head BP. Long-term atorvastatin treatment leads to alterations in behavior, cognition, and hippocampal biochemistry. Behavioural brain research 2014;267:6-11. [PubMed: 24657594]

65. Niethammer P, Delling M, Sytnyk V, Dityatev A, Fukami K, Schachner M. Cosignaling of NCAM via lipid rafts and the FGF receptor is required for neuritogenesis. J Cell Biol 2002;157(3):521532. [PubMed: 11980923]

66. Head BP, Peart JN, Panneerselvam M, Yokoyama T, Pearn ML, Niesman IR, Bonds JA, Schilling JM, Miyanohara A, Headrick J, Ali SS, Roth DM, Patel PM, Patel HH. Loss of caveolin-1 accelerates neurodegeneration and aging. PloS one 2010;5(12):e15697. [PubMed: 21203469] 


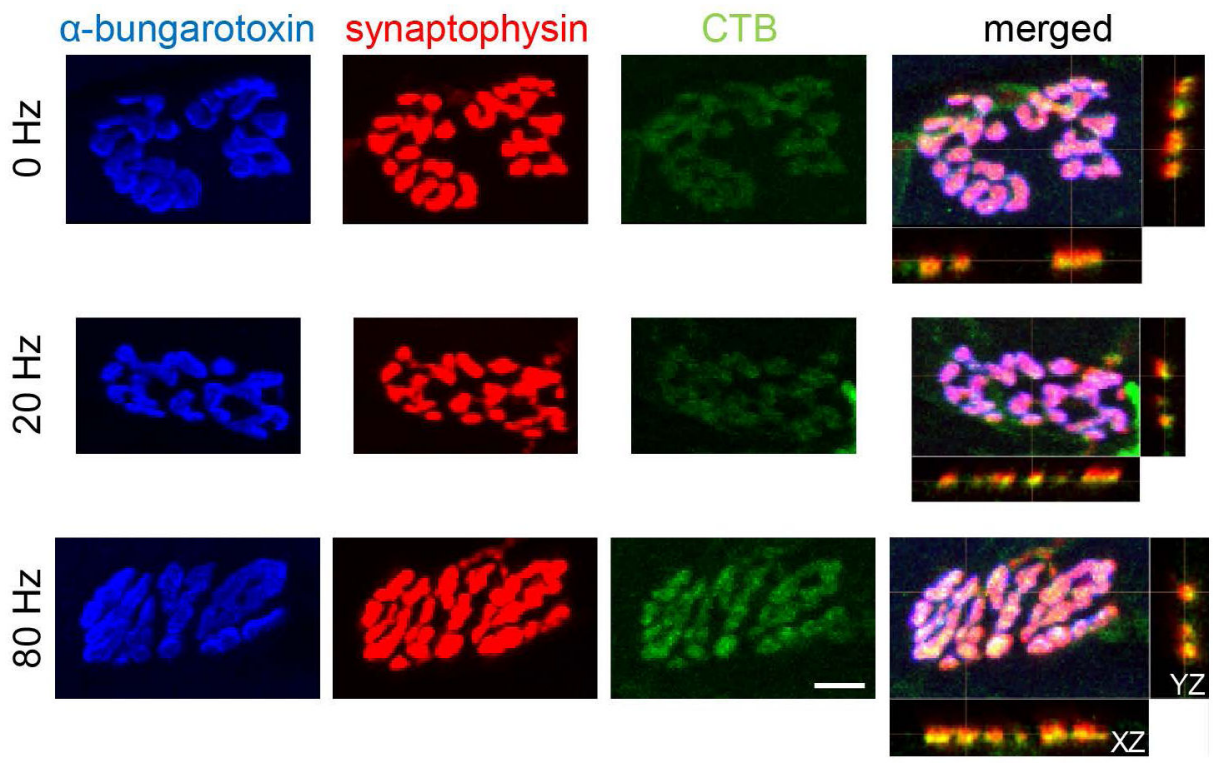

Figure 1.

Representative neuromuscular junctions (NMJ) in the rat diaphragm muscle obtained after phrenic nerve stimulation at various frequencies $(0,20,80 \mathrm{~Hz})$. Maximum intensity projections of fluorescent confocal images were obtained using post-synaptic acetylcholine receptors labeled by a-bungarotoxin, pre-synaptic axon terminals labeled by antisynaptophysin (a major synaptic vesicle protein), and lipid rafts labeled by cholera toxin subunit B (CTB); see Methods for details. The merged image with three fluorescent channels was used for volumetric analyses conducted in 3D. Orthogonal projections of an NMJ in each fiber type group show colocalization of synaptophysin (red) and CTB (green) in 3D. Synaptophysin and CTB partially overlap (yellow), and they are clearly present at the same anatomical structure. Scale Bar: $10 \mu \mathrm{m}$. 

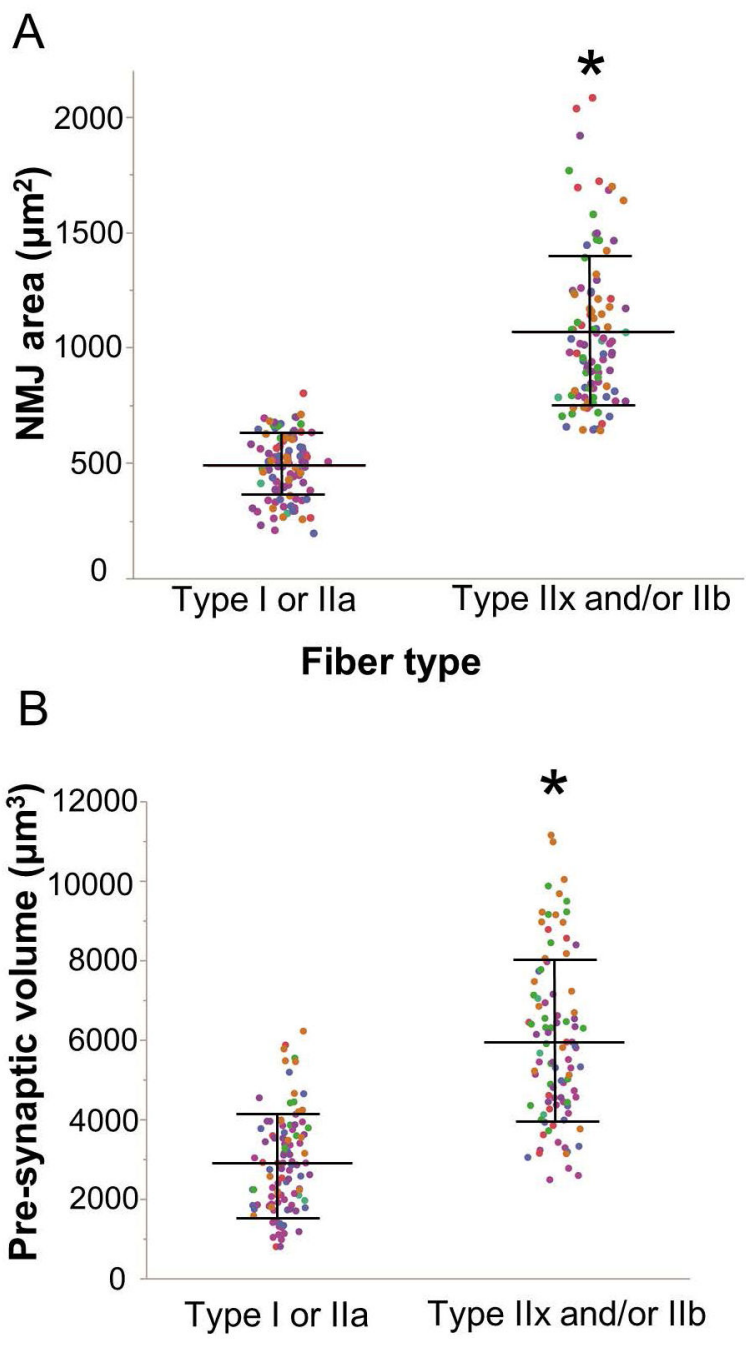

Fiber type

Figure 2.

Volumetric measurements of rat diaphragm NMJs at different muscle fibers types (classified according to established criteria of motor end-plate and NMJ complexity; see Methods for details). (A) Pre-synaptic volume is $~ 2$-fold larger at type IIx and/or IIb fibers, compared to type I or IIa fibers $(\mathrm{P}<0.0001)$. (B) Pre-synaptic volume is also 2-fold larger at type IIx and/or IIb fibers, compared to type I or IIa fibers ( $\mathrm{P}<0.0001)$. N=207 NMJs from 7 animals, with color denoting individual animals. Statistical significance was determined by two-way ANOVA with Tukey-Kramer HSD post-test. All values mean \pm SD. 
A

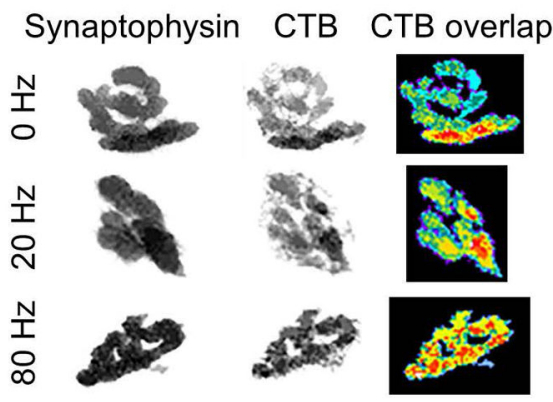

B
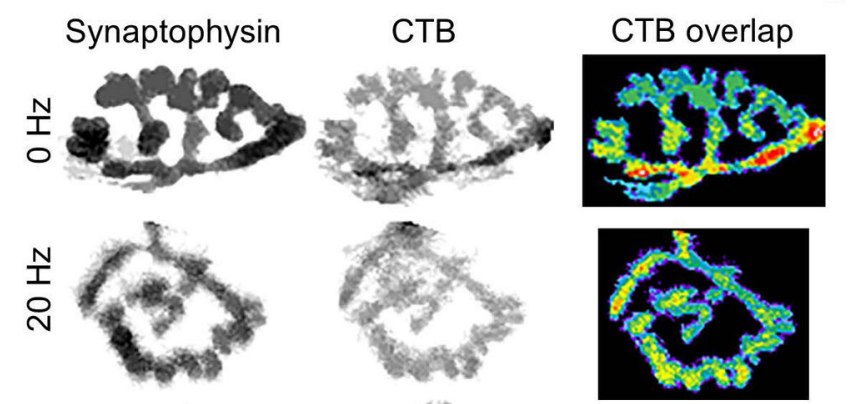

\section{C}
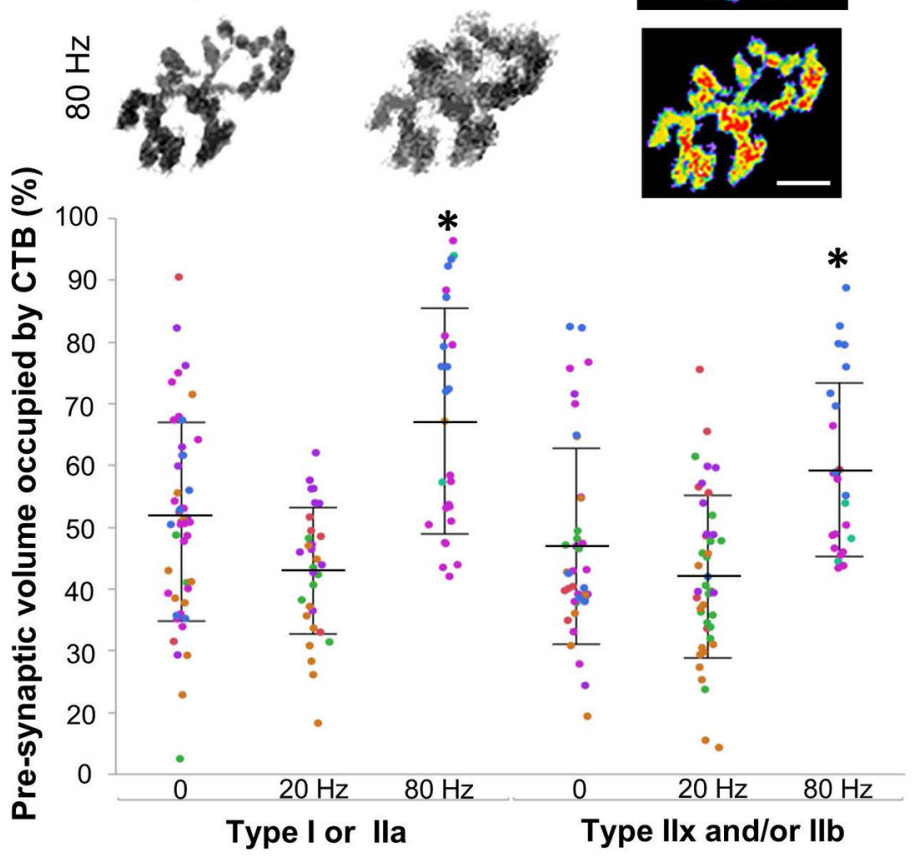

Figure 3.

Lipid raft uptake was quantified by the extent of CTB colocalization with synaptophysin at individual type-identified diaphragm NMJs. Representative three-dimensional (3D) reconstructions of the axon terminal volume (synaptophysin), lipid raft uptake (CTB) and the pre-synaptic volume occupied by lipid rafts (overlap) in axon terminals adjoining (A) type I or IIa fibers, and (B) type IIx and/or Ilb fibers, across various stimulation frequencies $(0,20$ and $80 \mathrm{~Hz})$. Images represent binarized masks for each confocal slice and display depth in gray and pseudocolored scales ( $\mu \mathrm{m}$; black and red-white reflect greater depth). (C) The fraction (\%) of pre-synaptic volume occupied by lipid rafts increased ( 20\%) at $80 \mathrm{~Hz}$ 
in axon terminals adjoining both diaphragm muscle fiber types $(\mathrm{P}<0.001)$. For NMJs at both muscle fiber types, there is not a significant difference in the percent of pre-synaptic terminal occupied by lipid rafts between the unstimulated and $20 \mathrm{~Hz}$ stimulated diaphragms ( $45 \%$ of axon terminal volume). N=207 NMJs from 7 animals (4 animals in $20 \mathrm{~Hz}$ group and 3 in $80 \mathrm{~Hz}$ ), with color denoting individual animals. Statistical significance was determined by two-way ANOVA with Tukey-Kramer HSD post-test. All values mean \pm SD. Scale bar $10 \mu \mathrm{m}$. 

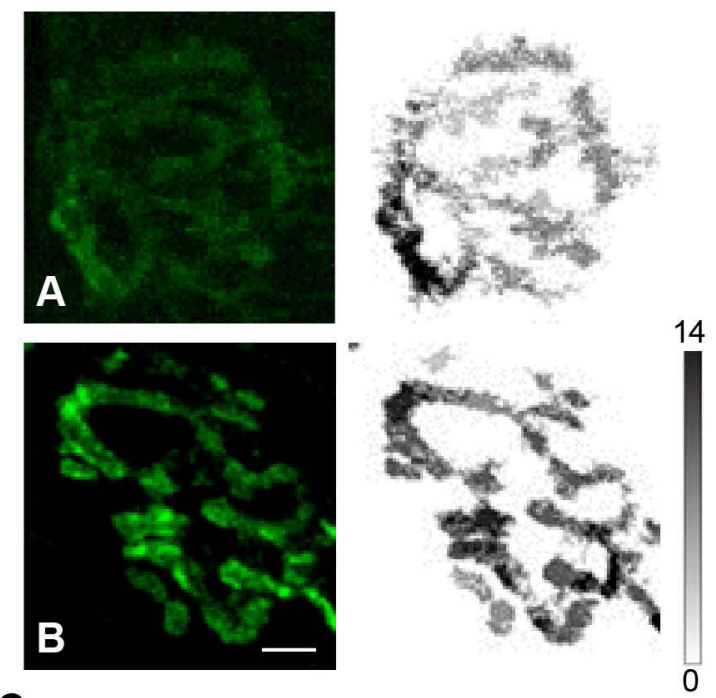

\section{C}

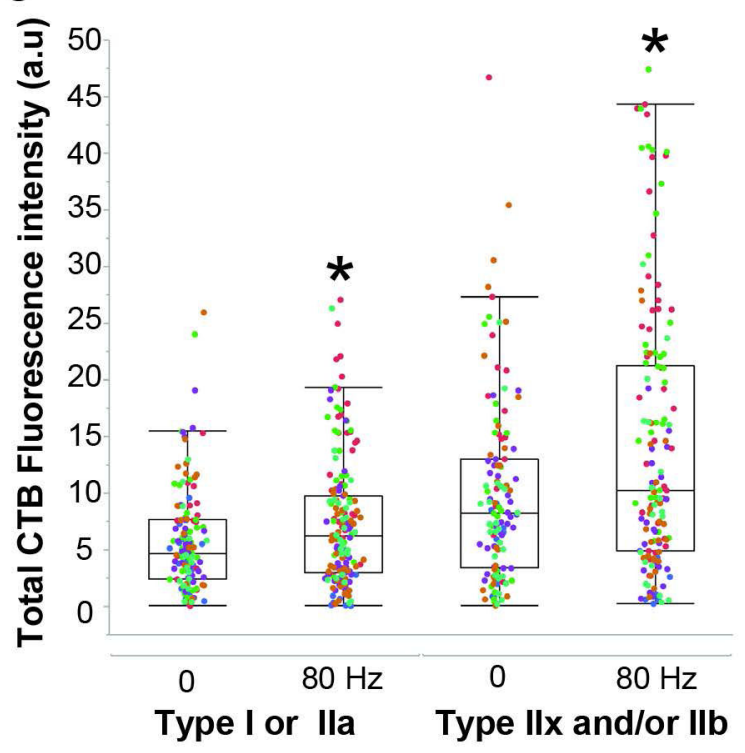

Figure 4.

Lipid raft uptake was quantified by total CTB fluorescence at type-identified rat diaphragm NMJs. Uptake increased after phrenic nerve stimulation at $80 \mathrm{~Hz}$ compared to no stimulation. Representative maximum intensity projections of CTB fluorescence (green) in presynaptic terminals of unstimulated (A) and $80 \mathrm{~Hz}$ stimulated (B) diaphragm musclephrenic nerve preparations. Representative three-dimensional reconstructions of CTB fluorescence comprise binarized masks for each confocal slice and display depth in gray scale ( $\mu \mathrm{m}$; black reflects greater depth). (C) In axon terminals at all types of NMJs, CTB intensity increased by $\sim 50 \%$ after $80 \mathrm{~Hz}$ stimulation $(\mathrm{P}=0.012$ for axon terminals adjoining type I or IIa fibers and $\mathrm{P}=0.003$ for type IIx and/or IIb fibers). Paired analyses were conducted for each animal since hemidiaphragms received either 0 or $80 \mathrm{~Hz}$ stimulation. $\mathrm{N}$ $=600$ NMJs from 6 animals, with color denoting individual animals. Statistical significance was determined by Wilcoxon test. All values mean \pm SD. Scale bar: $10 \mu \mathrm{m}$. 